\title{
Discovery of very low amplitude 9-minute multiperiodic pulsations in the magnetic Ap star HD 75445`
}

\author{
O. Kochukhov ${ }^{1}$, S. Bagnulo ${ }^{2}$, G. Lo Curto ${ }^{3}$, and T. Ryabchikova ${ }^{4,5}$ \\ 1 Department of Physics and Astronomy, Uppsala University, 75120 Uppsala, Sweden \\ e-mail: oleg@fysast.uu.se \\ 2 Armagh Observatory, College Hill, Armagh BT61 9DG, Northern Ireland, UK \\ 3 European Southern Observatory, Alonso de Cordova 3107, Vitacura, Santiago, Casilla 19001 Santiago 19, Chile \\ 4 Institute of Astronomy, Russian Academy of Sciences, Pyatnitskaya 48, 109017 Moscow, Russia \\ 5 Department of Astronomy, University of Vienna, Türkenschanzstraße 17, 1180 Vienna, Austria
}

Received 25 November 2008 / Accepted 8 December 2008

\section{ABSTRACT}

\begin{abstract}
We present our discovery of pulsational radial-velocity variations in the cool Ap star HD 75445, an object spectroscopically similar to the bright, rapidly-oscillating Ap (roAp) star $\gamma$ Equ. Based on high-resolution time-series spectroscopy obtained with the HARPS spectrometer at the European Southern Observatory 3.6-m telescope, we detected oscillations in Nd II and Nd III lines with a period close to 9 min and amplitudes of $20-30 \mathrm{~m} \mathrm{~s}^{-1}$. Substantial variation in the pulsational amplitude during our $3.8 \mathrm{~h}$ observing run reveals the presence of at least three excited non-radial modes. The detection of extremely low amplitude pulsations in HD 75445 indicates that the roAp excitation mechanism produces variability in the radial velocity amplitude of between a few tens $\mathrm{m} \mathrm{s}^{-1}$ and several $\mathrm{km} \mathrm{s}^{-1}$. This supports the idea that many, if not all, cool Ap stars occupying the roAp instability strip may harbour non-radial pulsations, which currently remain undetected due to their small photometric and radial-velocity amplitudes.
\end{abstract}

Key words. stars: atmospheres - stars: chemically peculiar - stars: oscillations - stars: individual: HD 75445

\section{Introduction}

Rapidly-oscillating Ap (roAp) stars are magnetic chemicallypeculiar stars pulsating in non-radial, magnetoacoustic $p$-modes of periods close to $10 \mathrm{~min}$. Pulsations in roAp stars are believed to be driven by the opacity mechanism operating in the hydrogen-ionization zone (Balmforth et al. 2001). The presence of strong magnetic fields in roAp stars enhances the driving of the high-overtone oscillations by the suppression of convection, influences pulsation frequencies, and determines the global geometry of the pulsational perturbations (Dziembowski \& Goode 1996; Saio 2005).

Strongly inhomogeneous vertical distributions of chemical elements combined with the rapid transformation of the outwardly-propagating pulsation waves are responsible for the unique spectroscopic pulsation signature of roAp stars (Ryabchikova et al. 2002). In particular, the lines of rare-earth elements (REEs) and the cores of the hydrogen lines pulsate with a factor of 10-100 higher amplitudes than the remaining spectral features (Kochukhov \& Ryabchikova 2001; Mkrtichian et al. 2003; Kurtz et al. 2006).

The observed roAp instability strip is limited to the $T_{\text {eff }}$ range 6400-8100 K, although theoretical stability calculations (Cunha 2002) predict pulsations in stars as hot as $9500 \mathrm{~K}$ and are unable to account for the unstable modes observed in stars with $T_{\text {eff }} \leq$ $7400 \mathrm{~K}$. At the same time, the coexistence of pulsating and apparently constant Ap stars in the same region of the H-R diagram has been a long-standing puzzle (e.g., Martinez \& Kurtz 1994).

\footnotetext{
* Based on observations collected at the European Southern Observatory, Chile (ESO programs 68.D-0254, 079.D-0118).
}

It is now understood that the time-resolved photometric techniques employed to detect and study variability in most of the 37 known roAp stars are insensitive to the low-amplitude pulsations observed in spectroscopic time-series analyses (Hatzes \& Mkrtichian 2004; Elkin et al. 2005). This leads to the suggestion that all magnetic Ap stars in a certain temperature range may oscillate, but some have amplitudes below the photometric detection threshold (Kochukhov et al. 2002).

We test this hypothesis by completing a high-precision survey of a sample of bright cool magnetic Ap stars using the High Accuracy Radial velocity Planet Searcher (HARPS) spectrograph at the European Southern Observatory (ESO). The first result of our observations - the discovery of 10.9-min oscillations in the Ap star HD 115226 - was reported by Kochukhov et al. (2008). Here we present the discovery of a new rapidly oscillating Ap star, HD 75445, which pulsates with one of the lowest radial-velocity (RV) amplitudes measured for roAp stars.

\section{Observations and data reduction}

We used the HARPS spectrograph (Mayor et al. 2003) at the ESO 3.6-m telescope at La Silla to monitor HD 75445 in the context of our search for low-amplitude variability in bright cool Ap stars (ESO program 079.D-0118). The star was observed on the night of April 15, 2007. The observations started at the barycentric JD 2454205.47129 and continued for $3.8 \mathrm{~h}$. We collected 120 consecutive $80 \mathrm{~s}$ exposures, separated by a dead time of $31 \mathrm{~s}$. The resulting time resolution of $111 \mathrm{~s}$ enabled us to detect variations with frequencies as high as $v=4.5 \mathrm{mHz}$ $(P=3.7 \mathrm{~min})$. 
The extraction of one-dimensional spectra and barycentric velocity correction of the wavelength scale was performed with the help of the HARPS pipeline. Our spectra have a nominal resolving power $R \equiv \lambda / \Delta \lambda=115000$, and cover a wavelength range from 3780 to $6910 \AA$, with a $30 \AA$ gap close to $5320 \AA$. Individual exposures of HD 75445 have peak signal-to-noise ratio of 70 per $15 \mathrm{~m} \AA$ pixel at $\lambda 6000 \AA$. In the final reduction step, one-dimensional extracted spectra of HD 75445 were postprocessed to achieve consistent continuum normalization following the procedure described in Kochukhov et al. (2007).

We did not employ the simultaneous ThAr method available at HARPS, avoiding contamination of the stellar signal. Instead, we acquired a ThAr reference spectrum at the beginning and end of the stellar observations. Using these calibrations, we estimated that the instrumental drift within the time series was below $0.1 \mathrm{~m} \mathrm{~s}^{-1}$. For the moderate signal-to-noise ratio of individual spectra of HD 75445, the dominant source of noise in the radial-velocity measurements was photon noise $\left(\geq 2 \mathrm{~m} \mathrm{~s}^{-1}\right)$ rather than the instrumental precision, which is similar to the measured drift.

\section{Basic properties of HD 75445}

The southern chemically-peculiar star HD 75445 (HIP 43257, $\left.\mathrm{CD}-38^{\circ} 4907\right)$ was classified as a Sr-Eu object by Bidelman \& MacConnell (1973). Its Strömgren photometric indices, $b-y=$ $0.159, m_{1}=0.218, c_{1}=0.729$ (Vogt \& Faundez 1979), $\mathrm{H} \beta=$ 2.801 (Maitzen et al. 2000), indicate $T_{\text {eff }}=7600-7700 \mathrm{~K}$ according to the calibrations by Moon \& Dworetsky (1985) and Napiwotzki et al. (1993). Geneva colours yield $T_{\text {eff }}=7680 \mathrm{~K}$ (Kochukhov \& Bagnulo 2006), in good agreement with the Strömgren photometry.

Kochukhov \& Bagnulo (2006) investigated the evolutionary state of HD 75445 using Hipparcos parallax and photometric $T_{\text {eff }}$. They determined $\log L=1.17 \pm 0.06 L_{\odot}, M=1.81 \pm$ $0.05 M_{\odot}$ and a stellar age that is a factor $0.56-0.72$ of the mainsequence lifetime. Ryabchikova et al. (2004) included HD 75445 in their abundance analysis study of a sample of roAp and nonpulsating Ap stars. Adopting $T_{\text {eff }}=7700 \mathrm{~K}$ and $\log g=4.3$, they showed that HD 75445 has close to solar Fe abundance, moderate enhancement of $\mathrm{Cr}$ and $\mathrm{Mn}, 1.6$ dex overabundance of $\mathrm{Co}$, and a large overabundance of several REEs. As for many known roAp stars, HD 75445 exhibits an ionization anomaly of Pr and $\mathrm{Nd}$, with doubly ionized lines of these elements providing an 1.3-2.0 dex higher abundance measurement than the lines of first ions. Ryabchikova et al. (2008) examined the Ca stratification and isotopic composition of HD 75445. They reported a 2.0 dex step-like change of the Ca concentration at $\log \tau_{5000}=$ -0.9 and detected the presence of heavy $\mathrm{Ca}$ isotopes $\left({ }^{46} \mathrm{Ca}\right.$ and ${ }^{48} \mathrm{Ca}$ ) in the upper atmospheric layers. This study also inferred a spectroscopic $T_{\text {eff }}=7650 \mathrm{~K}$ using the $\mathrm{H} \alpha$ line.

Ryabchikova et al. (2004) commented on the spectroscopic similarity of HD 75445 and the bright roAp star $\gamma$ Equ (HD 201601). This point is illustrated in Fig. 1 with new highquality, $R=115000$ spectra available for both stars (mean HARPS spectrum for HD 75445 and mean UVES spectrum derived from the archival time series data set of $\gamma \mathrm{Equ}$ ). The spectra of these two stars are almost identical, the only difference being slightly broader line profiles of $\gamma$ Equ due to the stronger mean surface field strength of this star. However, there is a discrepancy between the spectra of the two stars in the region of the resonance Li I doublet at $\lambda 6708 \AA$, which is strong in $\gamma$ Equ but entirely absent in HD 75445 (Kochukhov 2008).

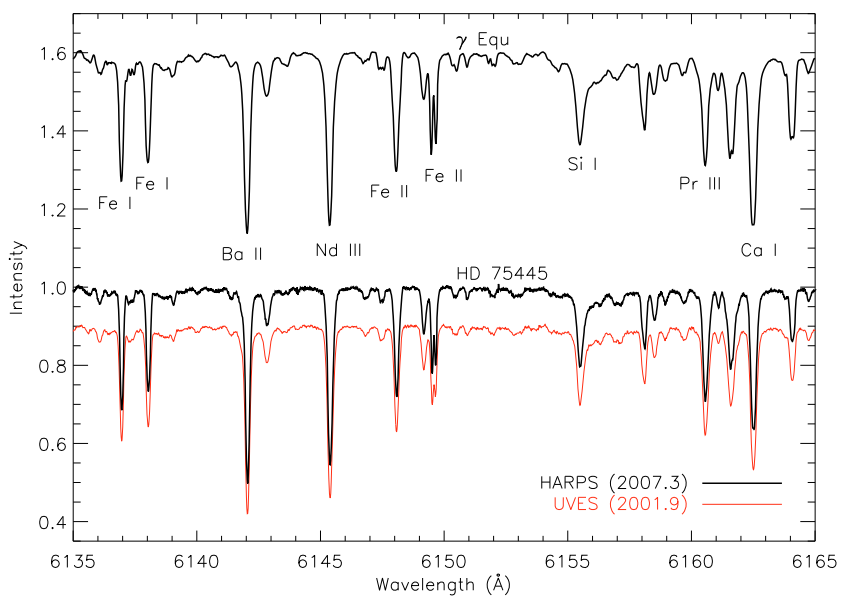

Fig. 1. Comparison of the 6135-6165 A region in the spectra of the new roAp star HD 75445 and the well-known bright roAp star $\gamma$ Equ. The UVES spectrum of $\gamma$ Equ is shown on top, with the identifications of the strongest spectral features. The mean HARPS spectrum of HD 75445 (middle, thick curve) is compared with the UVES observation of this star (bottom, thin curve) obtained 5.4 years before the HARPS observations. The UVES spectra are shifted vertically for display purposes.

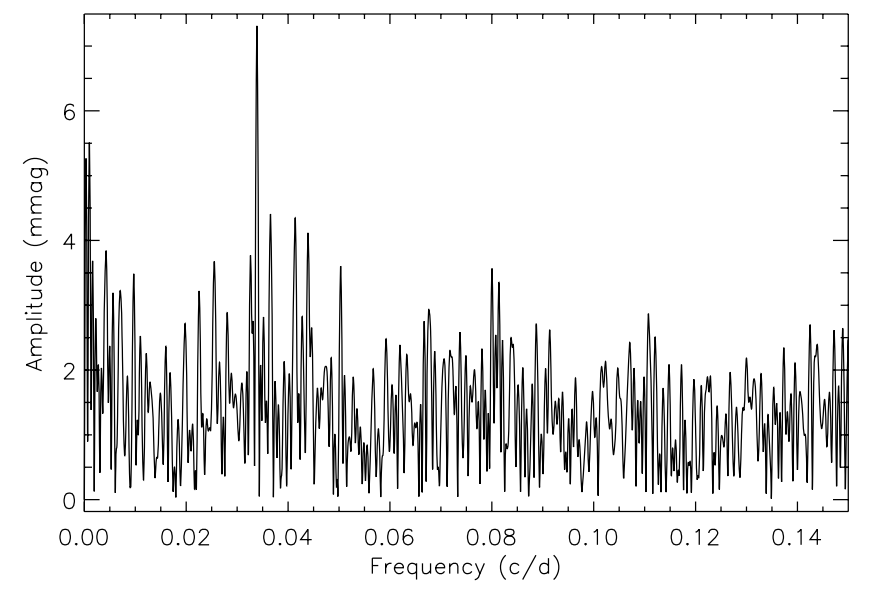

Fig. 2. Amplitude spectrum for the ASAS photometry of HD 75445 obtained after 2003.6.

Mathys et al. (1997) detected Zeeman splitting in the Fe II $6149 \AA$ line of HD 75445 and measured the mean field modulus $\langle B\rangle=2985 \pm 42 \mathrm{G}$ with 9 spectra recorded over the period of $450 \mathrm{~d}$ in 1994-1995. Ryabchikova et al. (2004) provided three additional measurements of $\langle B\rangle, 2915,2957$, and $2873 \mathrm{G}$, for the spectra obtained in 2000-2001. The splitting of Fe II $6149 \AA$ in our mean HARPS spectrum (2007.3) and in the UVES spectrum from 2001 (Ryabchikova et al. 2008) is consistent with $\langle B\rangle=$ $3030 \mathrm{G}$. In summary, the full set of $14\langle B\rangle$ measurements shows no evidence of periodic field strength variation.

We searched for long-period rotational brightness modulation in HD 75445 using the Hipparcos epoch photometry (ESA 1997) and the ASAS database (Pojmanski 2002). No periodic variability with an amplitude larger than 5 mmag was detectable in the Hipparcos light curve. The ASAS photometry of HD 75445 exhibited erratic brightness changes before 2003, which were probably instrumental in nature. Measurements obtained after 2003.6 did not deviate significantly from the mean value $V=7.14$. The amplitude spectrum computed for the ASAS observations of HD 75445 during 2003.6-2008.8 shows a marginal 7 mmag variability with a 29.5 d period (Fig. 2). 
This is consistent with our estimate of $v_{\mathrm{e}} \sin i \leq 2 \mathrm{~km} \mathrm{~s}^{-1}$ obtained by fitting profiles of magnetically insensitive Fe I lines at $\lambda$ 5434, 5576, and $5691 \AA$. Comparison of the mean HARPS spectrum with the UVES observation obtained 5.4 years before our observing run shows no detectable changes in the line profiles (Fig. 1), suggesting a very long rotation period.

Spectroscopic similarity of HD 75445 to $\gamma$ Equ, its prominent REE ionization anomaly, and its effective temperature of $T_{\text {eff }}<8000 \mathrm{~K}$ imply that this star is an obvious candidate for the search of oscillations (Ryabchikova et al. 2004). However, no photometric pulsation signature exceeding one mmag was detected for this star by Martinez (private communication). Here, we demonstrate that HD 75445 is indeed a roAp star but pulsating with an amplitude well below the current detection threshold of the ground-based, time-resolved photometry.

\section{Analysis of radial velocity variation}

We measured radial velocities of lines in the spectrum of HD 75445 using the centre-of-gravity technique (Kochukhov \& Ryabchikova 2001). Spectral line identification was based on the atomic line data extracted from the VALD database (Kupka et al. 1999), which includes the DREAM compilation of the REE line parameters (Biémont et al. 1999). The list of Nd III transitions was further extended using the study by Ryabchikova et al. (2006).

Previous time-resolved spectroscopic analyses of roAp stars (Kochukhov \& Ryabchikova 2001; Mkrtichian et al. 2003; Ryabchikova et al. 2007) demonstrated that maximum pulsation amplitudes are always found in singly and doubly ionized REE absorption features, such as Nd II, Nd III, and Pr III. A number of strong and medium-strength lines of REE ions are present in the spectrum of HD 75445. However, observational data available to us were of insufficiently high signal-to-noise ratio to detect pulsations in individual lines. We reduced the noise in the velocity curves by averaging RV measurements for all lines of a given REE ion. Among rare-earths, only Nd II and Nd III have sufficient number of lines in the spectrum of HD 75445 to yield precise combined RV measurements.

Using 29 lines of Nd III and 56 lines of Nd II, we achieved a noise level of 3-5 m s${ }^{-1}$ in the amplitude spectra and revealed conspicuous amplitude peaks in the $1.8-2.0 \mathrm{mHz}$ frequency range, which implied that oscillations of amplitude $20-30 \mathrm{~m} \mathrm{~s}^{-1}$ were present (Fig. 3, Table 1). These oscillation signatures are highly significant. The probability that noise would produce a peak of this observed amplitude at any frequency in the studied range (False Alarm Probability, Horne \& Baliunas 1986) is $7 \times 10^{-5}$ for Nd II and $4 \times 10^{-6}$ for Nd III. We also applied a bootstrap randomization technique (Kuerster et al. 1997), which is a more rigorous method of establishing the statistical significance of a peak in amplitude spectrum. Of the $10^{5}$ randomly shuffled data sets created from the original mean Nd II and Nd III RV curves, none exhibited spurious peaks of the observed amplitude in the frequency range $0-4.5 \mathrm{mHz}$. Thus, the probability that noise would create the signal detected in Nd II and Nd III lines is less than $10^{-5}$.

Applying a similar analysis procedure to 15 telluric lines in the $6275-6315 \AA$ region, we find no oscillations above $8 \mathrm{~m} \mathrm{~s}^{-1}$ with a noise level of $3 \mathrm{~m} \mathrm{~s}^{-1}$. Similarly, for the combined RV curve of $49 \mathrm{Fe}$ I and Fe II lines, which are unexpected to show significant variation in a low-amplitude roAp star, we found a maximum amplitude of $6 \mathrm{~m} \mathrm{~s}^{-1}$ and a noise level of $2 \mathrm{~m} \mathrm{~s}^{-1}$. The stability of telluric lines and the stellar Fe features confirms that
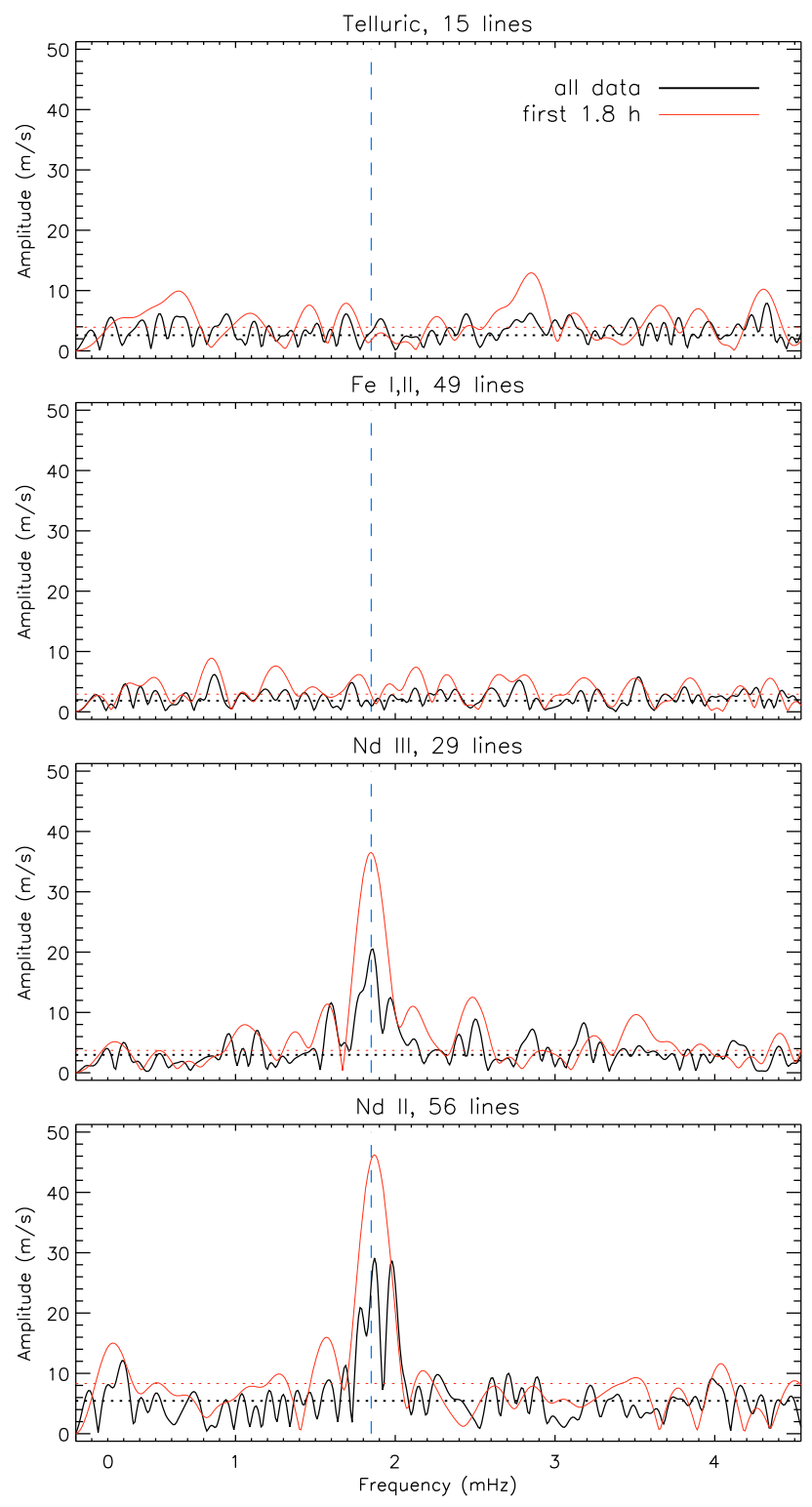

Fig. 3. From the top panel to the bottom panel: amplitude spectra for the average radial-velocity curves of 15 telluric lines, 49 lines of $\mathrm{Fe}$ I and II, 29 lines of Nd III, and 56 lines of Nd II. The vertical dashed line shows the main pulsation frequency $v=1.85 \mathrm{mHz}$. In each panel, the amplitude spectrum of the entire data set (thick curve) is compared to that of the first $1.8 \mathrm{~h}$ of the spectroscopic monitoring (thin curve). Horizontal dotted lines show corresponding noise levels.

variation detected in the Nd lines of HD 75445 is not due to an instrumental artifact.

The complex appearance of the Nd II and Nd III amplitude spectra in Fig. 3 suggests a multiperiodic pulsation. The presence of several excited modes in HD 75445 became apparent when the mean RV data was analysed in the time domain. We found that in the first 55 observations of HD 75445, corresponding to the initial $1.8 \mathrm{~h}$ of our time-resolved observations, pulsation variability was clearly evident. The amplitude spectra of this partial data set, illustrated in Fig. 3, indicated an almost monoperiodic pulsation. The least-squares fitting of this part of the oscillation curve yielded amplitudes of 36 and $46 \mathrm{~m} \mathrm{~s}^{-1}$ as well as pulsation periods of $8.93 \pm 0.05 \mathrm{~min}$ and $9.04 \pm 0.04 \mathrm{~min}$ for the singly and doubly ionized $\mathrm{Nd}$, respectively. There was a small lag of $0.44 \pm 0.19$ rad between the RV maxima of the two 
Table 1. Frequency analysis of the average radial velocity curves of telluric lines, Fe I and II, Nd III and Nd II. $N$ indicates the number of lines measured. $A_{\max }$ gives the highest radial velocity amplitude, followed by the estimate of False Alarm Probability of the corresponding signal. The last two columns give the amplitude of the variation with $v=1.85 \mathrm{mHz}$ $(P=9.01 \mathrm{~min})$ and the noise estimate.

\begin{tabular}{lccccc}
\hline \hline Ion & $N$ & $\begin{array}{c}A_{\max } \\
\left(\mathrm{m} \mathrm{s}^{-1}\right)\end{array}$ & FAP & $\begin{array}{c}A \\
\left(\mathrm{~m} \mathrm{~s}^{-1}\right)\end{array}$ & $\begin{array}{c}\sigma \\
\left(\mathrm{m} \mathrm{s}^{-1}\right)\end{array}$ \\
\hline Telluric & 15 & 7.9 & 0.31 & $2.5 \pm 2.5$ & 2.6 \\
Fe I,II & 49 & 6.2 & 0.31 & $1.5 \pm 1.8$ & 1.8 \\
Nd II & 56 & 29.1 & $6.6 \mathrm{E}-05$ & $24.6 \pm 4.8$ & 4.9 \\
Nd III & 29 & 20.5 & $\begin{array}{c}\text { 3.6E-06 } \\
\text { 20.7 20.4 } \pm 3.0\end{array}$ & 3.0 \\
& \multicolumn{5}{c}{ First 1.8 h } \\
Nd II & 56 & 46.2 & $8.7 \mathrm{E}-06$ & $44.2 \pm 5.5$ & 5.5 \\
Nd III & 29 & 36.5 & $1.1 \mathrm{E}-06$ & $36.4 \pm 3.8$ & 3.7 \\
\hline
\end{tabular}

Nd ions. In a similar way to other roAp stars (Ryabchikova et al. 2007), Nd III in HD 75445 showed a later maximum than Nd II.

The prominent sinusoidal variation in the Nd lines was subdued after about $2 \mathrm{~h}$ from the beginning of our observations, presumably due to beating of several excited modes as seen in other multiperiodic roAp stars (e.g., Sachkov et al. 2008). Tentative least-squares analysis suggested the presence of at least three significant frequencies: $v_{1}=1.81 \mathrm{mHz}\left(P_{1}=9.20 \mathrm{~min}\right), v_{2}=$ $1.85 \mathrm{mHz}\left(P_{2}=9.01 \mathrm{~min}\right)$, and $v_{3}=1.99 \mathrm{mHz}\left(P_{3}=8.37 \mathrm{~min}\right)$. The length of our time series does not allow $v_{1}$ and $v_{2}$ to be fully resolved. On the other hand, $v_{2}$ and $v_{3}$ were resolved, and $v_{3}$ was found to have a higher relative amplitude for $\mathrm{Nd}$ II.

\section{Discussion}

We have established the presence of multiperiodic pulsations in the cool magnetic Ap star HD 75445 using combined RV measurements of the lines belonging to Nd II and Nd III. The star exhibits oscillations with three frequencies, which have different amplitude ratios for the two Nd ions. The phase lag between RV curves of $\mathrm{Nd}$ II and Nd III can be interpreted in the framework of the outwardly propagating pulsational perturbation, which first reaches the layer where $\mathrm{Nd}$ II lines form and, after some delay, is seen in the higher atmospheric layer probed by stronger Nd III lines (Ryabchikova et al. 2007; Mashonkina et al. 2005). The difference in the amplitude ratios of the frequency components of the Nd II and Nd III RV curves can be ascribed to different vertical cross-sections of the three pulsation modes.

HD 75445 is the 38th known roAp star. Its discovery is significant because the star's pulsation amplitude is noticeably lower than for other roAp stars discovered to date using timeresolved spectroscopy. For example, HD 218994 (Gonzalez et al. 2007) and HD 115226 (Kochukhov et al. 2008) pulsate with an amplitude $\geq 500 \mathrm{~m} \mathrm{~s}^{-1}$, while for HD 116114 (Elkin et al. 2005) and HD 154708 (Kurtz et al. 2006) pulsations with an amplitude of $50-100 \mathrm{~m} \mathrm{~s}^{-1}$ were reported. Only for $\beta \mathrm{CrB}$ (HD 137909) comparable RV amplitudes of $20-30 \mathrm{~m} \mathrm{~s}^{-1}$ were found in individual lines of singly ionized REEs (Kurtz et al. 2007). However, $\beta \mathrm{CrB}$ is in many respects different from other roAp stars. It is an evolved star with a long pulsation period and a chemical composition deviating from that of a typical roAp star (Ryabchikova et al. 2004). In contrast, HD 75445 appears to have average roAp characteristics and is, in fact, a spectroscopic twin of the well-known roAp star $\gamma$ Equ. Nevertheless, it pulsates with an unusually low RV amplitude. This shows that although the atmospheric chemical composition, in particular the REE ionization anomaly, is helpful in selecting roAp candidates, it has no direct connection with the amplitude of oscillations in the line-forming region.

Kurtz et al. (2006) noted the tendency for weaker roAp oscillations to be found in stars with stronger fields. However, HD 75445 has a mean field modulus that is significantly weaker than many roAp stars but also shows an exceptionally low pulsation amplitude. We therefore conclude that a low-amplitude roAp pulsation can be present in cool Ap stars of any field strength. Although there are theoretical reasons to believe that the magnetic field alters the amplitude of the photospheric oscillations in few strong-field stars, a parameter other than the field intensity defines pulsation amplitude for other roAp stars.

The detection of very low amplitude pulsations in HD 75445 suggests that the roAp excitation mechanism produces oscillations with no apparent lower amplitude threshold. Thus, many cool Ap stars may possess pulsations with RV amplitudes $\ll 100 \mathrm{~m} \mathrm{~s}^{-1}$, which can be currently detected by time-resolved spectroscopy only in bright sharp-line stars such as HD 75445.

\section{References}

Balmforth, N. J., Cunha, M. S., Dolez, N., Gough, D. O., \& Vauclair, S. 2001, MNRAS, 323, 362

Bidelman, W. P., \& MacConnell, D. J. 1973, AJ, 78, 687

Biémont, E., Palmeri, P., \& Quinet, P. 1999, Ap\&SS, 269, 635

Cunha, M. S. 2002, MNRAS, 333, 47

Dziembowski, W. A., \& Goode, P. R. 1996, ApJ, 458, 338

Elkin, V. G., Riley, J. D., Cunha, M. S., Kurtz, D. W., \& Mathys, G. 2005, MNRAS, 358, 665

ESA 1997, The HIPPARCOS and TYCHO catalogues, ESA SP, 1200

Gonzalez, J. F., Hubrig, S., \& Savanov, I. 2007, Inform. Bull. Variable Stars, 5794, 1

Hatzes, A. P., \& Mkrtichian, D. E. 2004, MNRAS, 351, 663

Horne, J. H., \& Baliunas, S. L. 1986, ApJ, 302, 757

Kochukhov, O. 2008, A\&A, 483, 557

Kochukhov, O., \& Ryabchikova, T. 2001, A\&A, 374, 615

Kochukhov, O., \& Bagnulo, S. 2006, A\&A, 450, 763

Kochukhov, O., Landstreet, J. D., Ryabchikova, T., Weiss, W. W., \& Kupka, F. 2002, MNRAS, 337, L1

Kochukhov, O., Ryabchikova, T., Weiss, W. W., Landstreet, J. D., \& Lyashko, D. 2007, MNRAS, 376, 651

Kochukhov, O., Ryabchikova, T., Bagnulo, S., \& Lo Curto, G. 2008, A\&A, 479, L29

Kuerster, M., Schmitt, J. H. M. M., Cutispoto, G., \& Dennerl, K. 1997, A\&A, 320, 831

Kupka, F., Piskunov, N., Ryabchikova, T. A., Stempels, H. C., \& Weiss, W. W. 1999, A\&AS, 138, 119

Kurtz, D. W., Elkin, V. G., Cunha, M. S., et al. 2006, MNRAS, 372, 286

Kurtz, D. W., Elkin, V. G., \& Mathys, G. 2007, MNRAS, 380, 741

Maitzen, H. M., Paunzen, E., Vogt, N., \& Weiss, W. W. 2000, A\&A, 355, 1003

Martinez, P., \& Kurtz, D. W. 1994, MNRAS, 271, 129

Mashonkina, L., Ryabchikova, T., \& Ryabtsev, A. 2005, A\&A, 441, 309

Mathys, G., Hubrig, S., Landstreet, J. D., Lanz, T., \& Manfroid, J. 1997, A\&AS, 123,353

Mayor, M., Pepe, F., Queloz, D., et al. 2003, The Messenger, 114, 20

Mkrtichian, D. E., Hatzes, A. P., \& Kanaan, A. 2003, MNRAS, 345, 781

Moon, T. T., \& Dworetsky, M. M. 1985, MNRAS, 217, 305

Napiwotzki, R., Schoenberner, D., \& Wenske, V. 1993, A\&A, 268, 653

Pojmanski, G. 2002, Acta Astron., 52, 397

Ryabchikova, T., Piskunov, N., Kochukhov, O., et al. 2002, A\&A, 384, 545

Ryabchikova, T., Nesvacil, N., Weiss, W. W., Kochukhov, O., \& Stütz, C. 2004, A\&A, 423, 705

Ryabchikova, T., Ryabtsev, A., Kochukhov, O., \& Bagnulo, S. 2006, A\&A, 456, 329

Ryabchikova, T., Sachkov, M., Kochukhov, O., \& Lyashko, D. 2007, A\&A, 473, 907

Ryabchikova, T., Kochukhov, O., \& Bagnulo, S. 2008, A\&A, 480, 811

Sachkov, M., Kochukhov, O., Ryabchikova, T., et al. 2008, MNRAS, 389, 903

Saio, H. 2005, MNRAS, 360, 1022

Vogt, N., \& Faundez, A. M. 1979, A\&AS, 36, 477 